\title{
Analysis of Nuclear Power Station Control Signal and Alpha-Stable Noise Filter Method Based on LabVIEW
}

\author{
Xincai Chang, a, Jishi Guan ${ }^{1,2, b}$, Min Fu ${ }^{1, c}$ \\ ${ }^{1}$ China Nuclear Power Technology Research Institute Beijing Division \\ FL. $11^{\text {th }}$, CEC Building, No.6 south zhong guan cun St., Haidian District, Beijing, P.R.China \\ 2Jilin University, Nanling Campus 5988 Renmin StreetChangchun, Jilin, P.R.China

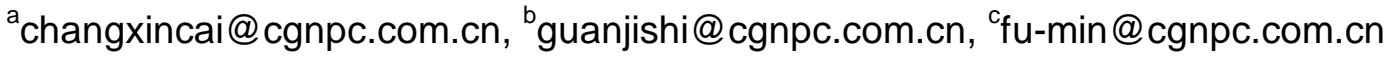

\begin{abstract}
Keywords: Alpha-Stable Noise, LabVIEW, Median Filter, Butterworth Filter
Abstract. This paper presents hardware in-the-loop simulation platform based on labview simulation, tests the response curve of nuclear power station control signal added the alpha-stable noise. Analysis of the response curve by labview signal processing module. By median filter and Butterworth Filter filtering process, compares the pros and cons of different filtering methods, chooses the best parameters, to achieve better filtering effect. Via signal after the matched filter effects, improves SNR, approaches the best performance of the system.
\end{abstract}

\section{Introduction}

As a kind of nonlinear filtering method, the median filter can eliminate the random noise well, especially for the isolated noise has a better effect [3][4]. Traditionally, mostly use Matlab and other software methods to achieve adaptive filter algorithm. In this paper, uses labview software signal processing module, chooses median filter function, modifies parameters by using median filter algorithm easily. Labview is a graphical language, referred to as "G" language, the simulation at the same time, simply add waveform display controls can be convenient to check the filtering effect, meet the requirements of real-time median filter.

Commonly used type of filter frequency response has three kinds of Butterworth, Bessel, and Chebychev. Butterworth filter is a kind of low pass filter with maximally flat amplitude response [1][2]; it has been widely used in communication field. Compared with the other two kinds of filter, butterworth low-pass filter due to the attenuation, phase characteristic and response characteristic has the advantages of equalization, so in actual use, butterworth filter has been widely used [7][8].

In the field of communication technology, the signal noise background is often used Gaussian distribution model to describe; this assumption is not accurate actually [9]. On atmospheric, sonar, electronic confrontation noise is similar to gaussian noise distribution of features, but has a thick tail [10]. This noise can be used to describe as alpha-stable distribution. This paper design in NI LabVIEW environment, build hardware in-the-loop simulation platform, alpha-stable noise simulated by computer. The nuclear power station control signal is controlled by NI-6259 card. The respond is acquisition and analysis by Labview. In LabVIEW, it provides a convenient man-machine interface; it is convenient for the user to select the control mode, the setting control parameters, and real-time acquisition and output waveform.

\section{Model Structure}

To overcome the flaws of the existing technology, this paper design a PI controller based on nuclear power station noise analysis device, hardware in-the-loop simulation platform is adopted to define the alpha-stable noise for the influence of the PI controller, provide data support for the alpha-stable noise eliminating.

As shown in Fig. 1 for the principle diagram of the control system of power station, including nuclear power station main box, and respectively connected to the alpha-stable noise simulator and the controller interface card. 


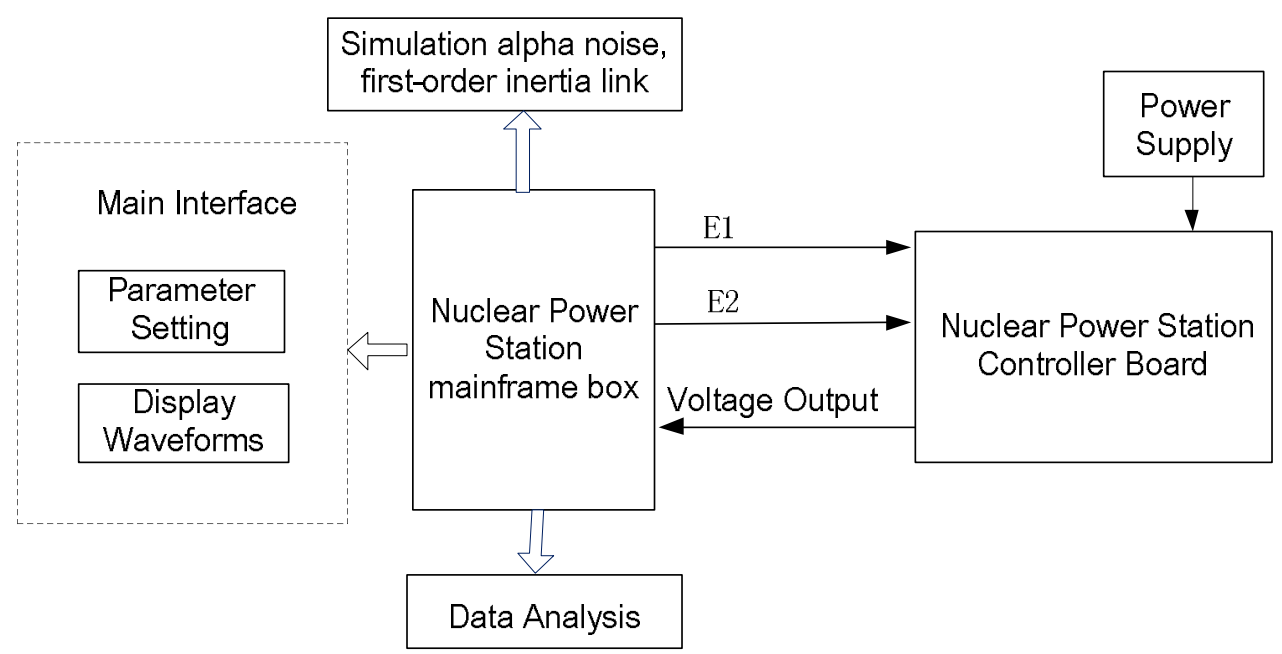

Fig. 1 model structure

The nuclear power station main box used to provide a platform and data forwarding. Users set up alpha-stable noise attribute information through this platform. The plant main box transmits alpha-stable noise attribute information to the alpha-stable noise simulator, to simulate and generate alpha-stable noise. Using standard $\mathrm{S} \alpha \mathrm{S}$ distribution model, the characteristic function is equations 1-4:

$$
\begin{aligned}
& \varphi(t)=\exp \left(j \alpha t-\gamma|t|^{\alpha}[1+j \beta \operatorname{sgn}(t) \omega(t, \alpha)]\right) \\
& \omega(t, \alpha)=\left\{\begin{array}{c}
\tan (\alpha \pi / 2), \alpha \neq 1 \\
(2 / \pi 1 \log |t|), \alpha=1
\end{array}\right. \\
& \operatorname{sgn}(u)\left\{\begin{array}{c}
1, u>0 \\
0, u=0 \\
-1, u<0
\end{array}\right\} \\
& -\infty<a<+\infty, \quad 0<\alpha \leq 2, \quad-1 \leq \beta \leq 1
\end{aligned}
$$

\section{LabVIEW Simulation}

The alpha-stable noise is simulated by NI-LabVIEW. Fig. 2-3 are the distributions of different alpha values of random variables in the sample sequence, and the corresponding response curves.

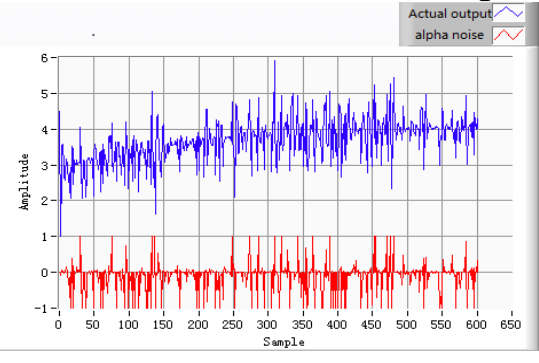

Fig. 2 alpha=0.6 response curve

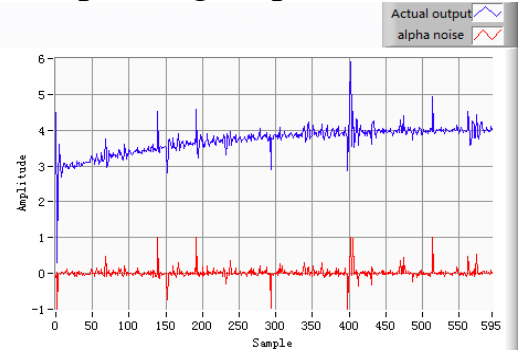

Fig. 3 alpha=1.2 response curve

Figure 4-7 are median filter and Butterworth filter Results with alpha=0.6and1.2 


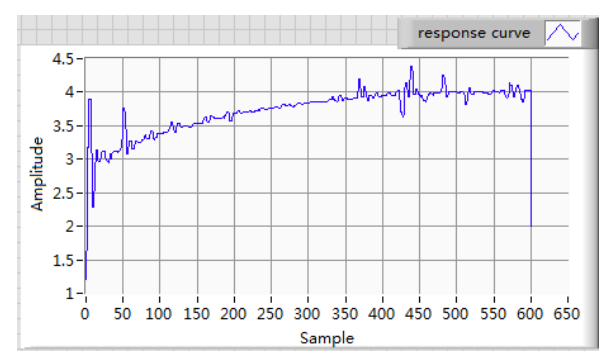

Fig. 4 alpha=0.6 median filter result

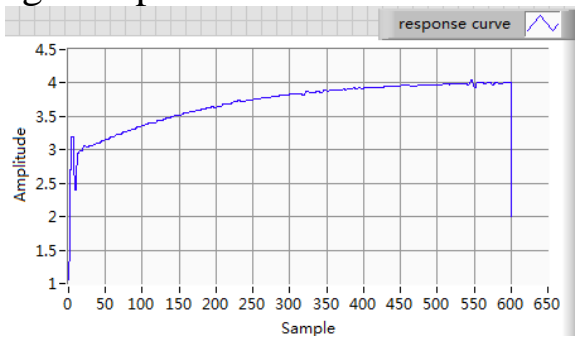

Fig. 6 alpha=1.2 median filter result

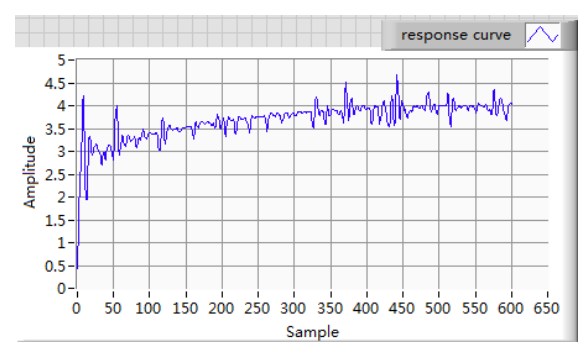

Fig. 5 alpha=0.6 Butterworth filter result

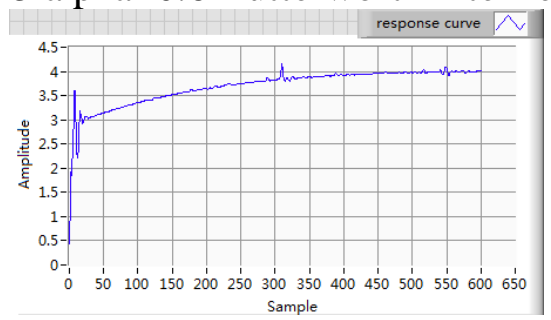

Fig. 7 alpha=1.2 Butterworth filter result

Fig. 8-9 are the two filter methods above combined with alpha=0.6and1.2

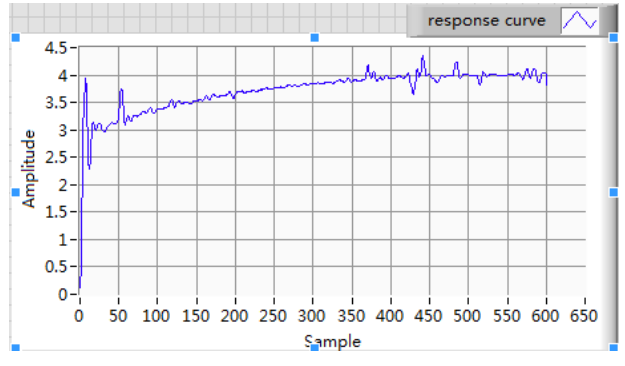

Fig. 8 alpha=0.6 two filter methods result

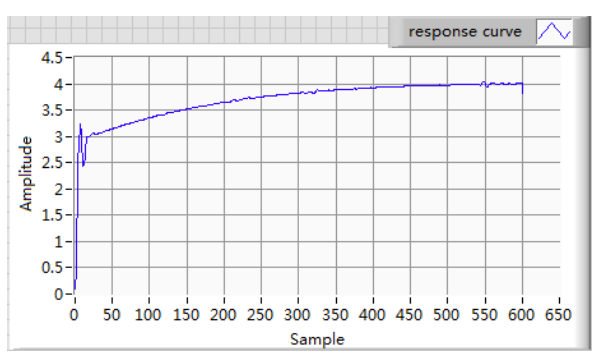

Fig. 9 alpha=1.2 two filter methods result

The LabVIEW simulation result show that the median filtering method and Butterworth filter method both can filter out part of alpha-stable noise, but can't completely filter, median filter method to the filtering effect of alpha-stable noise is superior to Butterworth filter. But will be a combination of the filtering effect is better.

\section{Conclusions}

In this paper, the alpha-stable noise is simulated by NI- LabVIEW software, using median filter and Butterworth filter of LabVIEW signal processing module respectively filtering system responses, comparative analysis of the two filtering processing result, and the two filtering methods combining processing system response. The results show that a simple median filter and Butterworth filter can not be perfect, a combination of both is better.

\section{References}

[1] Zhang Chengyun, Chen Minyi, Wang Jie; submitted to ICMTMA 2010

[2] Igor A. Litvin, Andrew Forbes; submitted to Optics Communications2008,281(9),2385-2392,8

[3] Vikas Gupta;Vijayshri Chaurasia ;Madhu Shandilya ; submitted to Journal of Visual Communication and Image Representation2015,26(000),296-304,9

[4] Shuang Ma ; Zhengxiang Shen; Shenghao Chen; Zhanshan Wang ; Optik - International Journal for Light and Electron Optics2015,126(24),4841-4845,5.

[5] Krishnan, S.R., Seelamantula, C.S.; in IEEE Transactions on Signal Processing2013,61(2),380-391,12. 
[6] Hamed Azami ; Karim Mohammadi ; Behzad Bozorgtabar; submitted to Journal of Signal and Information Processing2012,03(01),39-44,6

[7] Wazir Muhammad Laghari, Mohammad Usman Baloch, Muhammad Abid Mengal, Syed Jalal Shah ; submitted to Circuits and Systems2014,5(9),209-216,9

[8] S.N. Yao;T. Collins;Andrzej Odon; submitted to Computers \& Electrical Engineering2012,38(4),811-818,8 (2011)

[9] Navia-Vazquez, A.;Arenas-Garcia, J.; submitted to IEEE Transactions on Signal Processing2012,60(3),1478-1482,5

[10]Marco J. Lombardi; Simon J. Godsill in IEEE Transactions on Signal Processing2006,54(2),775-779,5 